\title{
MODULATION OF TEMOZOLOMIDE ACTION TOWARDS RAT AND HUMAN GLIOBLASTOMA CELLS IN VITRO BY ITS COMBINATION WITH DOXORUBICIN AND IMMOBILIZATION WITH NANOSCALE POLYMERIC CARRIER
}

\author{
N. S. FINIUK ${ }^{1}$, J. V. SENKIV ${ }^{1}$, A. O. RIABTSEVA ${ }^{2}$, \\ N. Y. MITINA ${ }^{2}$, N. I. MOLOCHII ${ }^{1}$, M. O. KITSERA ${ }^{1}$, \\ S. S. AVDIEIEV ${ }^{3}$, O. S. ZAICHENKO ${ }^{2}$, R. S. STOIKA ${ }^{1}$ \\ ${ }^{1}$ Institute of Cell Biology, National Academy of Science of Ukraine, Lviv; \\ ${ }^{2}$ Lviv National Polytechnic University, Ukraine; \\ ${ }^{3}$ Institute of Molecular Biology and Genetics, National Academy \\ of Science of Ukraine, Ukraine; \\ e-mail: stoika@cellbiol.lviv.ua
}

\begin{abstract}
Malignant gliomas (glioblastoma multiforme and anaplastic astrocytoma) occur more frequently than other types of primary central nervous system tumors, having a combined incidence of 5-8/100,000 population. Even with aggressive treatment using surgery, radiation, and chemotherapy, median reported survival is less than one year. Alkylating agents, such as temozolomide (TMZ), are among the most effective cytotoxic agents used for malignant gliomas, however, the responses still remain poor. Here, we present data about an enhancement of TMZ treatment effect towards rat and human glioma cells in vitro by immobilizing this drug with a new nanoscale polymeric-phospholipidic delivery system. It is a water-soluble comb-like poly(PM-co-GMA)-graft-PEG polymer consisting of a backbone that is a copolymer of 5-tert-butyl-peroxy5-methyl-l-hexene-3-yne (PM) and glycidyl methacrylate (GMA) and polyethylene glycol (PEG) side chains. The molecular weight of the carrier was $94,000 \mathrm{~g} / \mathrm{mol}$. Conjugation of TMZ with a novel polymeric carrier functionalized with phosphatidylcholine resulted in approximately 2 times enhancement of anticancer activity of TMZ. Combining of TMZ with doxorubicin $(50 \mathrm{nM})$ resulted in further enhancement by $23 \%$ of the antiproliferative effect of TMZ. TMZ caused apoptosis in glioma cells via activation of MAPK signaling pathway, inhibition of STAT3, and affected a transition through G2/M phase of cell cycle. These features make the novel nano-formulation of TMZ a perspective strategy for further development of this drug.
\end{abstract}

Key words: glioblastoma, apoptosis, temozolomide, doxorubicin, polymeric carrier.

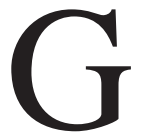
liomas (glioblastoma multiforme (GBM) and anaplastic astrocytoma (AA)) are the most common types of primary tumors of central nervous system (CNS) and have a combined incidence of 5-8/100,000 population. The five-year survival rates for brain tumors are the third lowest among all types of cancer where pancreas and lung cancers are the first and second, respectively [1]. The prognosis for patients with the advanced glioblastoma multiforme is very poor, and the mean survival period is 8-10 months. One of the challenges for testing new agents in this disease is the fact that brain tumors are uncommon, one-tenth as frequent as breast or lung cancer. Therefore, an unlimited number of large, prospective, randomized, controlled studies is not possible. As a result, there is reliance on nonrandomized studies as the principal design for identification of potentially active therapies that should be studied in more definitive, randomized trials [1].

The methylating drugs, such as temozolomide (TMZ), are widely used in treatment of brain tumors, particularly the malignant gliomas [2]. TMZ is a small (194 Da) lipophilic molecule that can be administered orally, and it crosses effectively the blood-brain barrier (BBB) [3]. TMZ is an imidazotetrazinone with activity attributed to the formation of a reactive methyldiazonium cation and methylation of O6-guanine in the DNA molecule. Clinical responses to TMZ are closely linked to the activity 
of O6-alkyl-guanine-DNA alkyl-transferase (AGT), a DNA repair protein that removes O6-alkyl-guanine adducts in the DNA [4]. TMZ advantages attractive for use in treatment of CNS tumors include excellent oral bioavailability and good circumvention of the BBB [5]. Although a survival for glioblastoma multiforme has not changed significantly over the past three decades, the emergence of novel treatment strategies for these tumors has led to heightened interest and optimism among oncologists [1]. New treatment strategies are emerging that target steps in the molecular pathogenesis of these tumors.

A perspective strategy to improve the activity of anticancer drugs is the combining of two or more drugs possessing different mechanisms of action that could produce the additive or synergistic effects. Generation of combinatorial approaches that act on the compensatory pathways in tumors may notably improve the effectiveness of the targeted therapy used alone [6].

New directions for the use of TMZ will likely focus on drug delivery to the targets in CNS. Since $90 \%$ of malignant gliomas recur within $1-2 \mathrm{~cm}$ of the original site, local therapy may be an effective strategy [1]. One of the methods here is the use of polymeric carriers to deliver drugs via diffusion from the micropores in the polymer matrix or by a release of drug within the interstices of a degradable matrix. Strategies to circumvent the BBB (polymers, bradykinin analogues, gene therapy) are important advances that have also shown an efficacy in early clinical trials. Future treatment strategies for malignant gliomas will likely involve synergistic combinations of agents aimed at different pathways in the molecular pathogenesis of this type of cancer.

A central approach is the development of the nanoparticle formulations to reduce the acute toxicity of free drug and improve its therapeutic efficacy. To reach that goal, various dendrimer-, polymer-, liposome-, and micelle-based systems were designed as delivery vehicles $[7,8]$. Besides, a decrease of adverse effects, several nanoparticle-based drug delivery systems $[9,10]$ were developed in order to bypass specific efflux proteins and to selectively increase drug accumulation in drug-resistance tumor cells.

In this article, we developed the conjugate of TMZ with novel polymeric carrier (A24-p-TMZ) functionalized with the phosphatidylcholine. We aimed to examine whether such modification of TMZ can enhance its treatment effect towards rat and human malignant glioma cells grown in vitro. The mechanisms of the proapoptotic action of TMZ in free and polymer-immobilized forms have been evaluated.

\section{Materials and Methods}

Cell lines and culture conditions. The C6 rat glioma and T98G human glioblastoma multiform cell lines were obtained from a collection at the Institute of Molecular Biology and Genetics, National Academy of Sciences of Ukraine (Kyiv, Ukraine). Cell lines were cultured in Dulbecco's modified Eagle's medium (DMEM, Sigma-Aldrich, USA) supplemented with $10 \%$ fetal bovine serum (APP, Austria). Cells were cultivated in the $\mathrm{CO}_{2}$-thermostate at $37{ }^{\circ} \mathrm{C}$ in atmosphere of $95 \%$ air and $5 \% \mathrm{CO}_{2}$ at $100 \%$ humidity.

Reagents. Temozolomide (TMZ, 3,4-dihydro-3-methyl-4-oxoimidazo [5,1-d]-as-tetrazine8-carboxamide, Fig. 1) was kindly supplied by the Department of Functional genomics (Institute of Molecular Biology and Genetics, National Academy of Sciences of Ukraine). Doxorubicin (Dox) was purchased from Arterium (Ukraine) via a standard pharmacy distribution.

The polymeric carrier (Fig. 2) was synthesized at the Department of Organic Chemistry at Lviv Polytechnic National University (Lviv, Ukraine). It was a water-soluble comb-like polymer poly(PM-coGMA)-graft-PEG consisting of backbone that was copolymer of 5-tert-butyl-peroxy-5-methyl-l-hexene3-yne (PM) and glycidyl methacrylate (GMA) and polyethylene glycol (PEG) side chains. The molecular weight of the carrier was $94.000 \mathrm{~g} / \mathrm{mol}[11,12]$. To prepare TMZ delivery system, solution of $100 \mathrm{mg} / \mathrm{ml}$

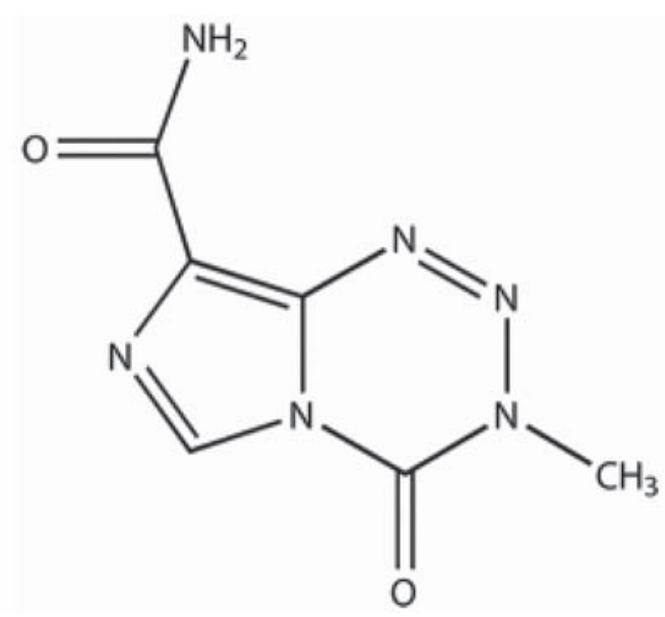

Fig. 1. Temozolomide 


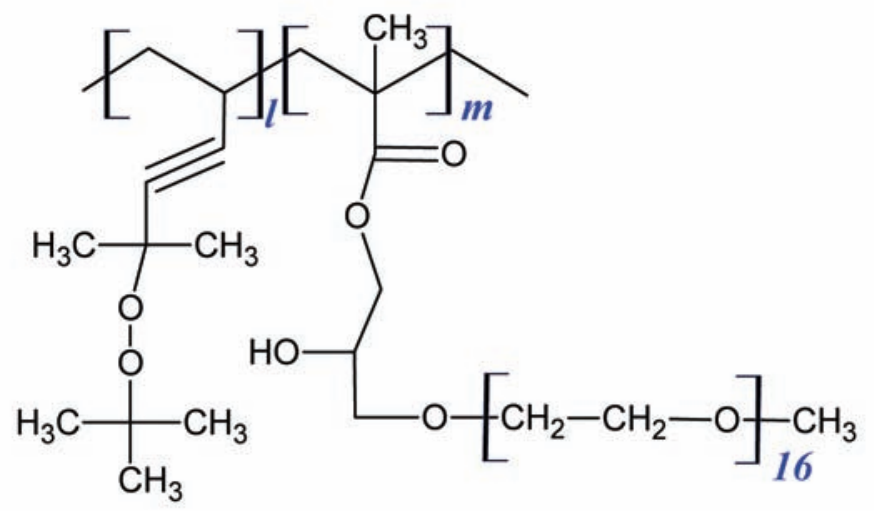

Fig. 2. Structure of the of the polymeric carrier functionalized with the phosphatidylcholine

poly(PM-co-GMA)-graft-PEG was prepared in the dimethyl sulfoxide (DMSO, Sigma-Aldrich, USA). For preparation of phosphatidylcholine, containing system $50 \mathrm{mg}$ of phosphatidylcholine were dissolved in $1 \mathrm{ml}$ of chloroform, then chloroform was evaporated to obtain thin film of the phosphatidylcholine. $100 \mathrm{mg} / \mathrm{ml}$ solution of poly(PM-co-GMA)-graftPEG in DMSO was added to the phosphatidylcholine film and kept at room temperature for $1 \mathrm{~h}$. Then, $3.0 \mathrm{mg}$ of TMZ were dissolved in $0.5 \mathrm{ml}$ DMSO and the resulting solutions of TMZ and polymer were mixed and added drop by drop to $8.5 \mathrm{ml}$ water containing $0.9 \% \mathrm{NaCl}$. Finally, the solution was stirred for $1 \mathrm{~h}$ and then sonicated for $20 \mathrm{sec}$.

Anti-proliferative assays. Screening of anticancer activity in vitro of free TMZ and its encapsulated form with the polymeric carrier functionalized with the phosphatidylcholine and Dox (the last was used here as a reference drug control) was measured using MTT assay [13]. Tumor cells were grown for $24 \mathrm{~h}$ in 96-well plates ( $0.1 \mathrm{ml})$ at 5,000 cells/well. After that, cells were incubated for $72 \mathrm{~h}$ with various additions of the synthesized compounds (final concentration of $0 ; 50 ; 100 ; 250 \mu \mathrm{M})$. MTT reagent which is converted to dark blue, water insoluble MTT formazan by the mitochondrial dehydrogenases, was used to determine viable cells according to the manufacturer's protocol (Sigma-Aldrich, USA). The $\mathrm{IC}_{50}$ of the tested compounds was calculated as a lethal concentration of drug killing 50\% cells in comparison with an untreated cell culture. The viability of the untreated cells was regarded as $100 \%$.

Fluorescent microscopy. FITC-conjugated Annexin V and Propidium Iodide (PI) double staining were performed in order to detect early apoptotic events in C6 rat glioma cells treated with free and conjugated with polymeric carrier TMZ, and polymeric carrier. FITC-conjugated Annexin V (SigmaAldrich, USA) and PI (Sigma-Aldrich, USA) were added directly to the culture medium of drug-treated C6 rat glioma cells to reach a final concentration of $250 \mu \mathrm{M}$ of free and polymeric carrier functionalized with phosphatidylcholine, polymer-encapsulated TMZ, and A24-p-TMZ. In $72 \mathrm{~h}$ after the addition of the tested compound, the C6 cells were pelleted by centrifugation at 2,000 rpm, washed twice with $1 \mathrm{x}$ Phosphate Buffered Saline (PBS), and incubated for 15 min in the Annexin V binding buffer containing 1/20 volume of FITC-conjugated Annexin V solution and PI $(20 \mu \mathrm{g} / \mathrm{ml}) .10 \mu 1$ of cell suspension were added on slides and cover glasses were placed over them. The cells were examined under Zeiss AxioImager A1 fluorescent microscope (Carl Zeiss, Germany).

Western blot analysis. After $48 \mathrm{~h}$ exposure of the tested compound, cell proteins were isolated, resolved by SDS/PAGE, and transferred onto a polyvinylidene difluoride (PVDF) membrane for Western blotting, as described [14]. The following antibodies were used at a 1:1,000 dilution: anti-ERK $1 / 2$, antiSTAT3, anti-phospho-cdc2 (Tyr 15) (Cdk1), antiphospho-Rb (Ser 807/811), anti-cleaved Caspase 3 (Cell Signaling Technology, USA), anti-JNK (sc571), anti-Cdk2 (sc-6248) (Santa Cruz Biotec., USA). Equal protein loading of each lane was evaluated by the immunoblotting of the same membrane with anti-beta-actin monoclonal mouse AC-15 (Sigma-Aldrich, USA). All secondary peroxidase-labelled antibodies (Cell Signaling, USA) were used at working dilution of 1:5,000. 
Statistical analysis. All data are presented as mean \pm SD. Results were analyzed and illustrated with GraphPad Prism (version 6; GraphPad Software, San Diego, CA). Statistical analyses were performed using two-way ANOVA with Bonferroni post-tests (apoptosis induction, tumor growth). A $P$ value of $<0.05$ was considered statistically significant.

\section{Results and Discussion}

Anticancer activity of temozolomide. To examine the antitumor effect in vitro of free TMZ and its complex with the polymeric carrier functionalized with the phosphatidylcholine (A24-p-TMZ) towards glioma cells, we treated glioma cell lines (rat C6 and human T98G) with $0-250 \mu \mathrm{M}$ of studied compounds for $72 \mathrm{~h}$. Then, cell viability was determined by the MTT assay.

When chemo-sensitivity of C6 rat glioma cell line towards studied compounds was studied, similar dose-dependent changes in cellular viability was found. The $\mathrm{IC}_{50}$ (concentration resulting in cell viability of $50 \%$ of control) of TMZ was $74.6 \pm 5.1 \mu \mathrm{M}$, while the IC $_{50}$ of A24-p-TMZ was $72.4 \pm 4.1 \mu \mathrm{M}$ (Table). However, T98G human glioblastoma multiform cells showed to be more resistant to TMZ $\left(\mathrm{IC}_{50}=243.1 \pm 3.2 \mu \mathrm{M}\right)$ than to the A24-p-TMZ $\left(\mathrm{IC}_{50}=165.9 \pm 3.1 \mu \mathrm{M}\right)$ (Table).

It should be noted that the polymeric carrier functionalized with phosphatidylcholine itself did not affect proliferation of $\mathrm{C} 6$ and T98G glioma cells considerably (Fig. 3).

As shown in Fig. 4, 5, TMZ inhibited viability of glioma cells in a dose- and time-dependent man-

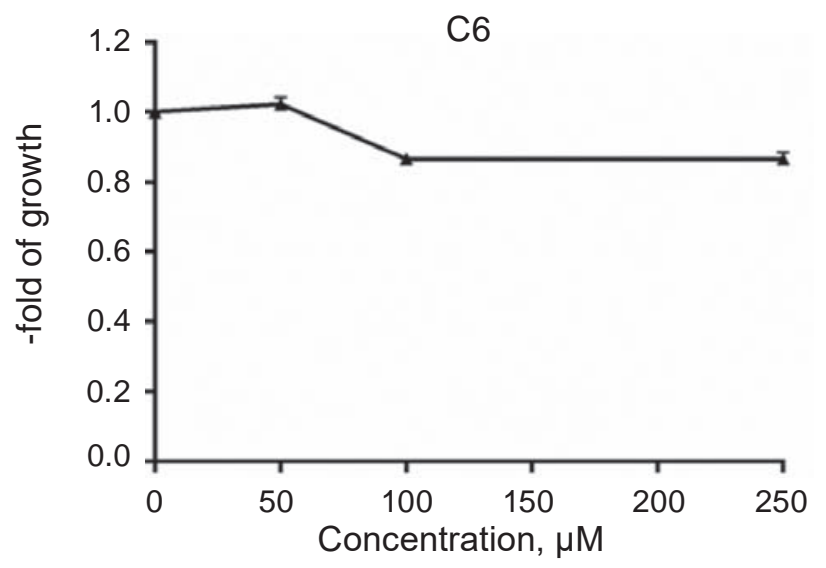

Cytotoxic effect of free and polymeric carrier encapsulated temozolomide

\begin{tabular}{|c|c|c|}
\hline \multirow{2}{*}{ Cell line } & TMZ & A24-p-TMZ \\
\hline & $\mathrm{IC}_{50} \pm \mathrm{SD}, \mu \mathrm{M}$ & $\mathrm{IC}_{50} \pm \mathrm{SD}, \mu \mathrm{M}$ \\
\hline C6 rat glioma & $74.6 \pm 5.1$ & $72.4 \pm 4.1$ \\
\hline $\begin{array}{l}\text { T98G human } \\
\text { glioblastoma } \\
\text { multiform }\end{array}$ & $243.1 \pm 3.2$ & $165.9 \pm 3.1$ \\
\hline
\end{tabular}

ner. The cytotoxity of free TMZ and its form encapsulated by the polymeric carrier functionalized with the phosphatidylcholine towards glioma cells was carried out in 3, 24, 48 and $72 \mathrm{~h}$. It was found that $\mathrm{IC}_{50}$ value of A24-p-TMZ complex in case of treating C6 cell line was reached as soon as in $3 \mathrm{~h}$ of incubation (Fig. 4). While the $\mathrm{IC}_{50}$ value at the action of free TMZ was reached only in $48 \mathrm{~h}$ of incubation. In $6 \mathrm{~h}$ of incubation, the cytotoxic effects of A24-p-TMZ complex and free TMZ were almost the same. We have observed difference in the cytotoxicity of drugs after $24 \mathrm{~h}$ and $48 \mathrm{~h}$ incubation, where A24-p-TMZ complex possessed higher cytotoxic effect at lower concentration of used substances. However, similar $\mathrm{IC}_{50}$ of free and conjugated form of TMZ towards C6 cell line were found (Fig. 4).

Next, we studied the cytotoxic effect of free and polymer-conjugated form of TMZ, and the polymeric carrier towards T98G human glioblastoma multiform cell line (Fig. 5).

We have found that free TMZ achieved its $\mathrm{IC}_{50}$ at $48 \mathrm{~h}$ treatment of human T98G glioblastoma mul-

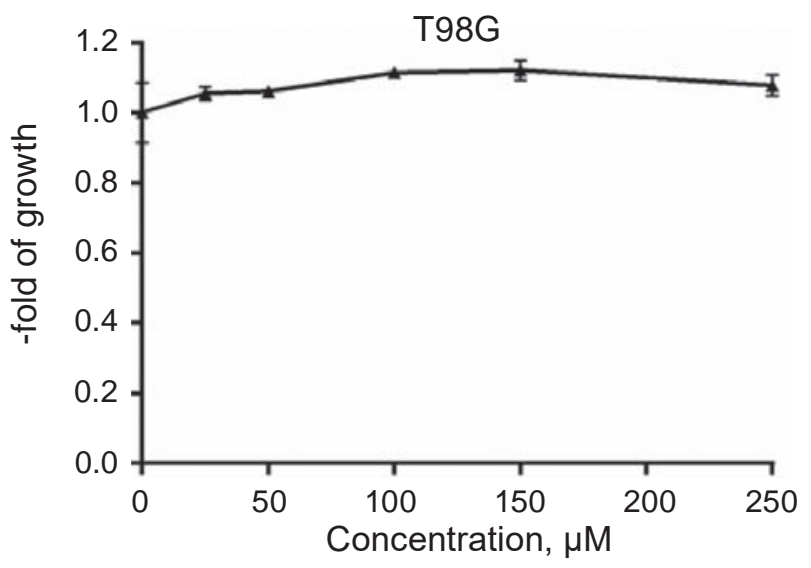

Fig. 3. Comparison of cytotoxicity of the polymeric carrier functionalized with the phosphatidylcholine (A24-p) towards different glioma cell lines. After a total experimental time of $72 \mathrm{~h}$, cell vitality was measured by the MTT assay 

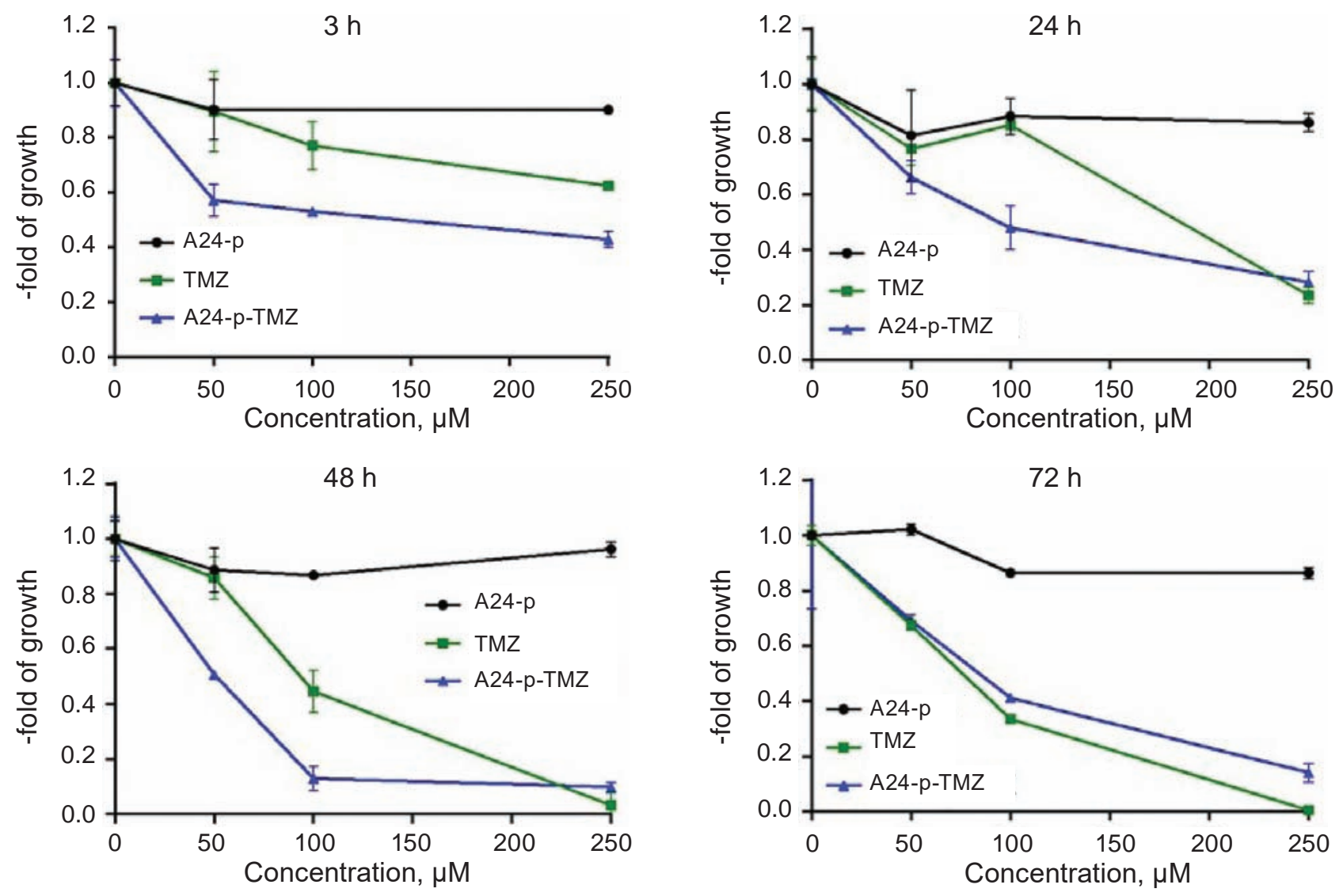

Fig. 4. Exposure time-dependency of the action of free temozolomide and its form encapsulated by the polymeric carrier functionalized with the phosphatidylcholine towards C6 rat glioma cells. Measurements were performed after 3, 24, 48 and $72 \mathrm{~h}$ exposition to compounds. Viability of cells was determined using MTT assay

tiform cells $\left(\mathrm{IC}_{50}=245.7 \pm 2.4 \mu \mathrm{M}\right)$. After $72 \mathrm{~h}$ of incubation, the $\mathrm{IC}_{50}$ was $243.14 \pm 3.21 \mu \mathrm{M}$ that is almost 3 times higher value than the $\mathrm{IC}_{50}$ for rat $\mathrm{C} 6$ glioma cells $\left(\mathrm{IC}_{50}=74.55 \pm 5.06 \mu \mathrm{M}\right)$. TMZ conjugated with the polymeric carrier functionalized with the phosphatidylcholine reached its $\mathrm{IC}_{50}$ at $48 \mathrm{~h}$ of incubation. The $\mathrm{IC}_{50}$ of the conjugated form of TMZ was $165.9 \pm 3.1 \mu \mathrm{M}$ that is 1.5 times lower than that of the free form of TMZ.

Thus, conjugation of TMZ with novel polymeric carrier functionalized with the phosphatidylcholine leads to enhancement of the antiproliferative activity of this drug.

The synergistic effect of doxorubicin combined with free and conjugated forms of temozolomide. Combination of two or more drugs possessing different mechanisms cytotoxic action or demonstrating different mechanisms of circumventing drug resistance can produce additive or synergistic effects in the action of anticancer drugs [6]. That is why, to improve the antitumor effect in vitro of free and conjugated form of TMZ, we have additionally used traditional anticancer drug - the Dox. To investigate possible synergistic effect of Dox in combination with various forms (free and encapsulated) of TMZ, we used MTT assay after $72 \mathrm{~h}$ of incubation of cells with the studied drugs.

The obtained results demonstrated that the presence of Dox in cultural medium did not affect the antitumor action of free form of TMZ (Fig. 6). However, Dox increased the cytotoxic action of TMZ conjugated with the polymeric carrier functionalized with the phosphatidylcholine towards rat C6 glioma cells. The addition of Dox ( $50 \mathrm{nM})$ to the culture medium led to a decrease in the value of $\mathrm{IC}_{50}$ from $67.9 \mu \mathrm{M}$ to $52.46 \mu \mathrm{M}$. So, the effectiveness of A24-p-TMZ complex was improved by $23 \%$ with its combination with the Dox.

Mode of temozolomide action. Previously, it was reported that TMZ-induced apoptosis in the 

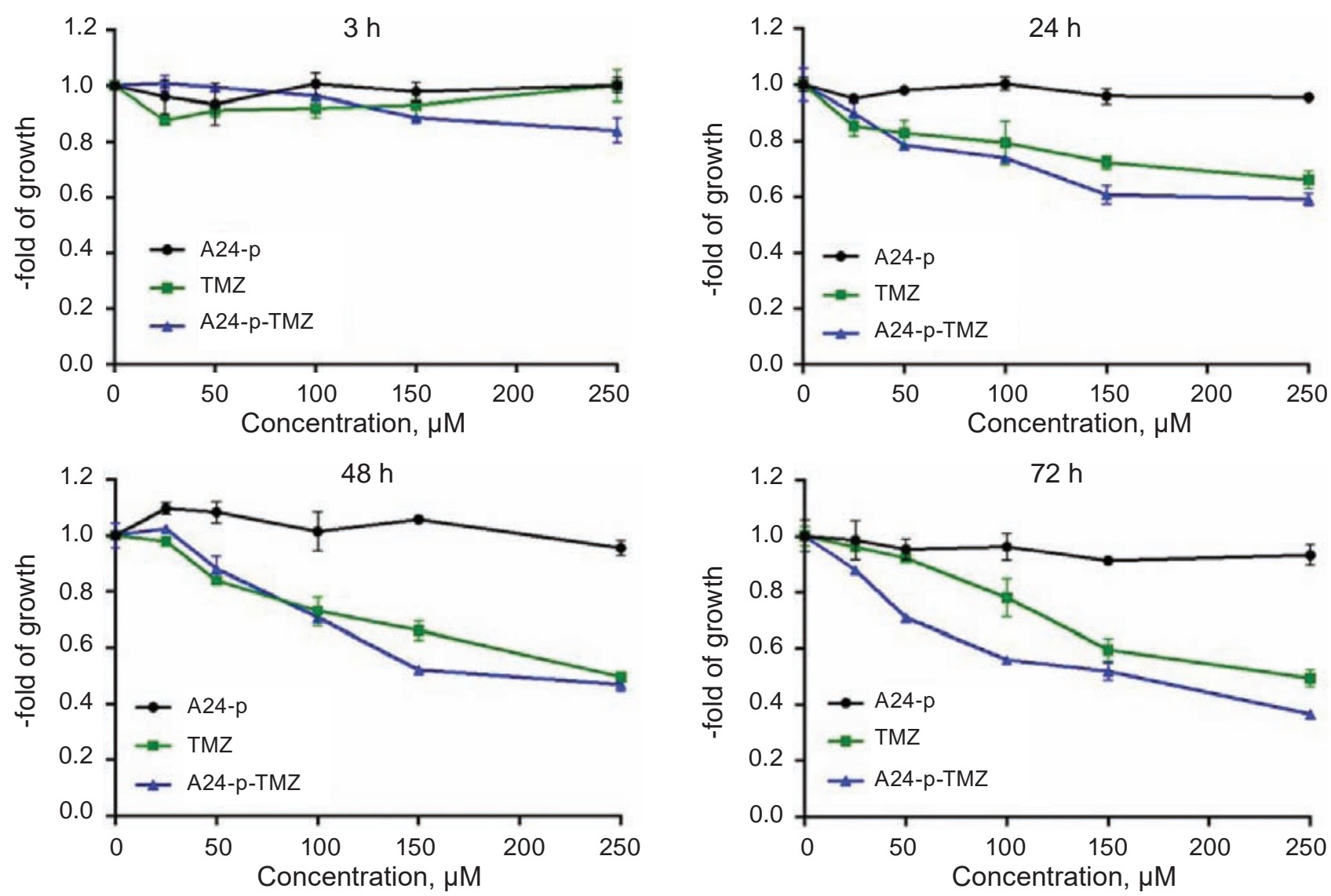

Fig. 5. Exposure time-dependency of free temozolomide and its form encapsulated by the polymeric carrier functionalized with the phosphatidylcholine towards T98G human glioblastoma multiform cell line. Measurements were performed after 3,24, 48 and $72 \mathrm{~h}$ of exposition to studied compounds. Viability of cells was determined using MTT assay
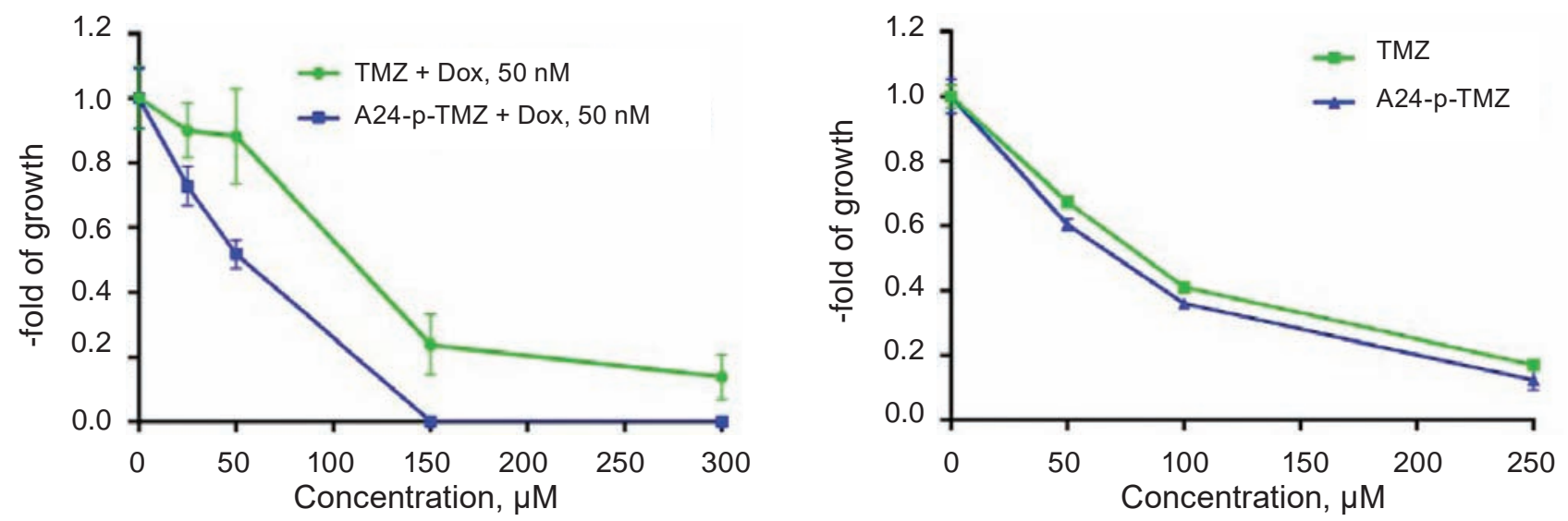

Fig. 6. Patterns of cytotoxic action of free temozolomide and its form conjugated with the polymeric carrier functionalized with the phosphatidylcholine towards rat C6 glioma cells under addition of $50 \mathrm{nM}$ doxorubicin. After a total experimental time of $72 \mathrm{~h}$, cell vitality was measured by the MTT assay

glioblastomas, however precise mechanisms of such action are still not clearly understood [15]. To determine whether TMZ-treated glioma cells un- dergo apoptosis, we performed a supra-vital double staining with FITC-conjugated Annexin V and PI of rat glioma cells treated with free TMZ and its form 


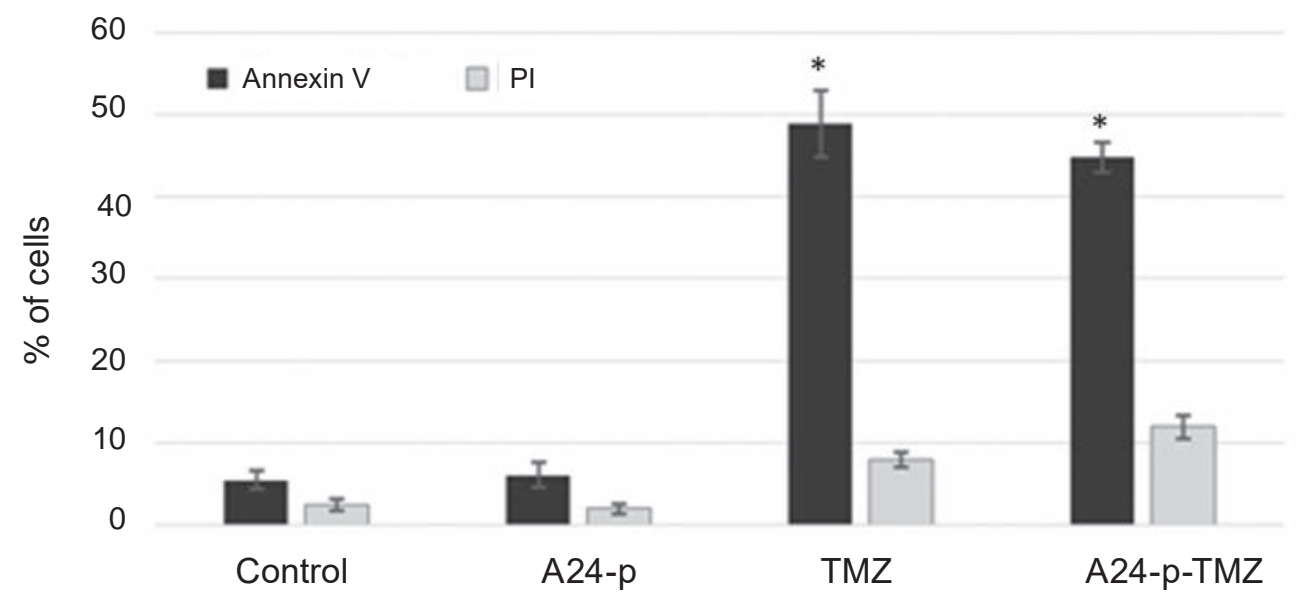

Fig. 7. Ratio of dead cells treated for $72 \mathrm{~h}$ with free temozolomide and its form conjugated with the polymeric carrier functionalized with the phosphatidylcholine. Ratio of dead cells was determined by the Annexin $V$ (indicator of apoptosis) and PI (indicator of necrosis) co-staining of rat C6 glioma cells. Percentage of the apoptotic cells was analyzed using the fluorescent microscopy

conjugated with the polymeric carrier functionalized with the phosphatidylcholine. As shown in Fig. 7, $48.9 \%$ of apoptotic cells were detected in rat C6 glioma cells treated for $72 \mathrm{~h}$ with TMZ $(75 \mu \mathrm{M})$. While the polymer-conjugated form of TMZ induced appearance of $44.8 \%$ apoptotic C6 glioma cells. Thus, the cytotoxic action of TMZ is due to induction of apoptosis in these cells.

At the next stage of study, we explored the signaling pathways for apoptosis during action of free and conjugated forms of TMZ in combination of Dox. The reason to conduct such study was that we have shown that low dose of Dox (50 nM) enhanced the cytotoxic action of polymer-conjugated form of TMZ (see: Fig. 6), while other investigators found that Dexamethasone decreased temozolomideinduced apoptosis in human gliobastoma T98G cells [15].

It is known that cellular stresses and some other stimuli can induce apoptosis via modulating a signaling pathway of the mitogen-activated protein kinases (MAPK) [16]. Here we found that conjugated TMZ enhanced phosphorylation of JNK kinases. A combination with Dox (50 nM) of free TMZ and its conjugated form with the polymeric carrier also caused an activation of JNK kinases (Fig. 8). These proteins are activated as a result of negative impact of some external factors. In turn, pJNK induces activation of a large number of specific proteins involved in apoptosis (p53, ATF-2, c-Jun and others). Thus, an increase in the amount of pJNK could be a characteristic feature of cells that die by apoptosis.
Treatment of C6 glioma cells with TMZ conjugated with the polymeric carrier caused a reduction in the amount of STAT3 protein. A combination of TMZ conjugated with polymeric carrier with Dox also caused a decrease in STAT3 protein amount. Free form of TMZ and combination of Dox with TMZ did not affect the amount of STAT3 protein (Fig. 8). According to literature data, the phosphorylated STAT3 is a transcription activator acting as a signaling molecule for many cytokines and growth factors. This protein was activated at different types of cancer and possessed an oncogenic effect and anti-apoptotic properties [16].

We have also found an increased amount of CDK2 protein under the action of TMZ conjugated with the polymeric carrier and under the action of combination of Dox with free TMZ and its conjugated form with the polymeric carrier (Fig. 8).

Next, we studied the mechanism of the proapoptotic action of free TMZ and its polymerconjugated form in combination with the Dox in human T98G glioblastoma cells. It was found that the amount of cleaved Caspase 3 increased more definitely under the effect of free form of TMZ than under the effect of polymer-conjugated TMZ. However, the combination of TMZ with Dox decreased the level of cleavage of Caspase 3 (Fig. 9).

The obtained results also showed an increase of JNK kinase in T98G cells under the effect of all forms of TMZ and its combination with the Dox $(50 \mathrm{nM})$. Besides, we have found that free TMZ and its form conjugated with the polymeric carrier inde- 


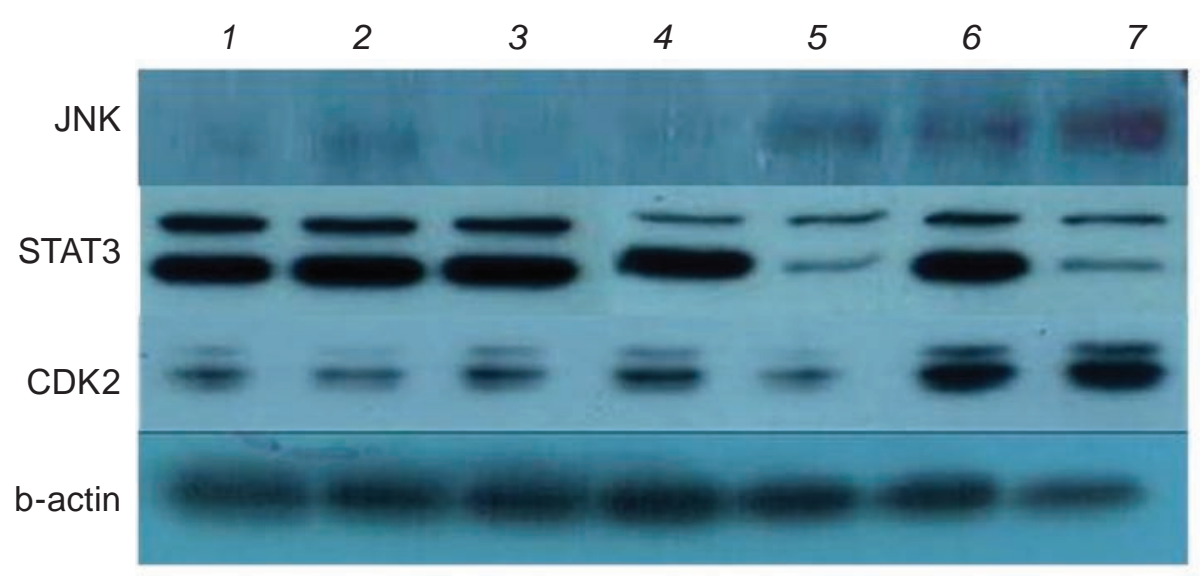

Fig. 8. Pattern of expression of the pro-apoptotic and cell cycle regulation proteins in T98G cells after their treatment for $24 \mathrm{~h}$ with free temozolomide and its form conjugated with the polymeric carrier functionalized with the phosphatidylcholine. 1 - Control; 2 - polymer A24-p (300 $\mu M)$; 3 -Dox (50 nM); 4-A24-p-TMZ $(300 \mu M) ; 5-T M Z(300 \mu M) ; 6-A 24-p-T M Z(300 \mu M)$ combined with the Doxorubicin (50 nM); $7-T M Z$ $(300 \mu M)$ combined with the Doxorubicin (50 nM)

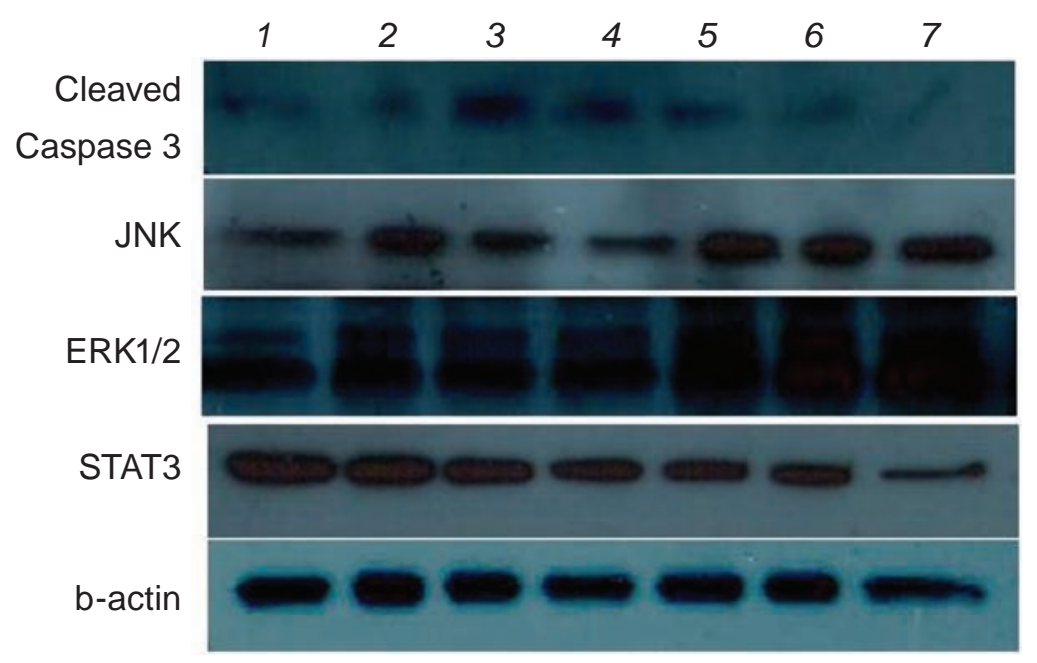

Fig. 9. Pattern of expression of the pro-apoptotic proteins in human T98G glioblastoma cells treated for $24 \mathrm{~h}$ with free temozolomide and its form conjugated with the polymeric carrier functionalized with the phosphatidylcholine. 1 - control; 2 - polymer A24-p (250 $\mu M)$; 3 -Dox (50 nM); 4 - TMZ (250 $\mu M)$; 5 - A24p-TMZ (250 $\mu M) ; 6$ - TMZ (250 $\mu M)$ combined with Doxorubicin (50 nM); 7 - A24-p-TMZ (250 $\mu M)$ combined with Doxorubicin (50 $\mathrm{nM}$ )

pendent on a combination with the Dox caused an increase in the amount of ERK 1/2 protein (Fig. 9).

Treatment of the T98G glioma cells with free TMZ and its form conjugated with the polymeric carrier independent on a combination with the Dox caused a reduction in the amount of STAT3 protein. Combination of Dox with TMZ conjugated with the polymeric carrier caused more pronounced decrease in the amount of STAT3 protein (Fig. 9).
Combination with Dox of TMZ conjugated with the polymeric carrier decreased an amount of cyclin-dependent kinase 1 (Cdk1). It is known that an elevation of the Cdk1 plays a key role in controlling the eukaryotic cell cycle through modulating centrosome cycle, as well as a mitotic onset. Cdk1 promotes the G2/M transition and regulates G1 progress and the G1-S transition via association with the multiple interphase cyclins [17]. 
While the amount of phosphorylated form of $\mathrm{Rb}$ protein under the effect of TMZ alone was rather weak, TMZ combined with the Dox caused more pronounced increase in the amount of phosphorylated $\mathrm{pRb}$. Although, combination of Dox with TMZ conjugated with the polymeric carrier did not affect the level of phosphorylated pRb (Fig. 10).

Thus, the obtained results demonstrated that cytotoxicity of TMZ towards glioma cells is related to induction of their apoptosis via activation of MAPK signaling pathway, inhibition of STAT3, and affecting a transition of $\mathrm{G} 2 / \mathrm{M}$ phase in cell cycle.

Patients suffering from malignant glioma, in particular glioblastoma multiforme, have a very poor prognosis. Standard therapy, in addition to surgery and radiotherapy, includes treatment with alkylating agents, specifically TMZ, and the chloroethylating drugs, such as carmustine and nimustine. In future, the use of TMZ as well as other methylating drugs is expected to become more dominant than a use of chloroethylating agents because of less severe negative side effects [2].

Application of the nanocarriers for delivery of anthracyclines including the Dox can be a successful strategy aimed at improvement of the pharmacological characteristics and, thus, at reduction of adverse effects. Most of available nanoparticles were designed for addressed tumor targeting. In this study, we present a novel polymeric/phospholipid conjugate of TMZ which resulted in an enhanced anticancer activity in vitro. It was found that nanocomplexation of TMZ increased approximately twice its antipro- liferative activity. It could be due to the selectively increase of the drug accumulation in tumor cells and slow drug release into the interstitial space of the tumor [18, 19]. Application of such kind of complex stimulates the cells to accumulate the drug by endocytosis pathway and by macropinocytosis pathway. Endocytotic drug uptake could be the promising strategy to diminish the effects of chemotherapy resistance [20]. In addition, many nanoparticles that are PEGylated are characterized by reduced binding to serum proteins or (immune) cells and, such, evade their degradation via incorporation by macrophages [18].

The mechanism of apoptosis induction by the O6-methylating agents has been elucidated in detail in various experimental models such as rodent cell lines [21], lymphoblastoid cells [22] and peripheral human lymphocytes [2]. However, this mechanism remains enigmatic in the malignant gliomas and some other tumor types. It was reported that in human glioma cells TMZ might induce either a p53-associated G2/M arrest followed by cell senescence or p53-independent mitotic catastrophe [23]. In another study, TMZ was proposed to induce an autophagy, while it failed to induce apoptosis in the malignant glioma cells [3], although glioma cells growing as spheriods were shown to be sensitive to apoptosis induced by the alkylating agent [24].

These contradictory data prompted us a necessity of studying in more detail the mechanism of induction of glioma cell death upon treatment with TMZ. Here, we demonstrated that glioma cells un-

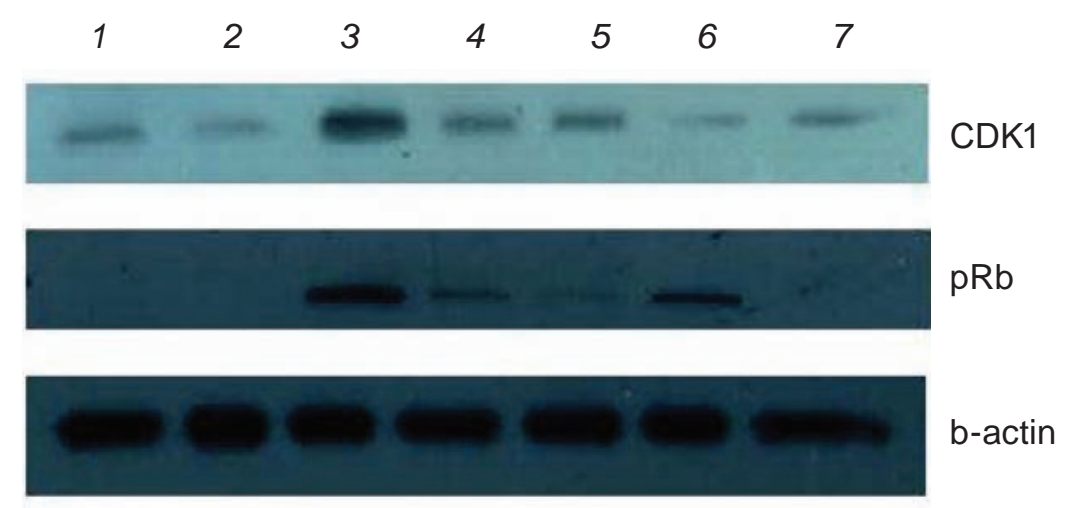

Fig. 10. Pattern of expression of the pro-apoptotic and cell cycle regulation proteins in human T98G glioblastoma cells treated for $24 \mathrm{~h}$ with free temozolomide and its form conjugated with the polymeric carrier functionalized with the phosphatidylcholine. 1 - control; 2 - polymer A24-p (250 $\mu M) ; 3$-Dox (50 nM); 4 -TMZ (250 $\mu M) ; 5$ - A24-p-TMZ (250 $\mu M) ; 6-T M Z(250 \mu M)$ combined with Doxorubicin (50 nM); 7 - A24-p-TMZ $(250 \mu M)$ combined with Doxorubicin (50 nM) 
dergo apoptosis following their treatment with free TMZ and its form conjugated with the polymeric carrier functionalized with the phosphatidylcholine.

Other strategy aimed at enhancement of the activity of anticancer drugs is the combining the action of two or more drugs. Favorable safety profile of TMZ allows it to be co-administered with various additional agents. The antitumor activity of TMZ is dependent on the level of alkyl-guanine alkyltransferase (AGT) in tumor cells. The results of the preclinical studies indicate that inhibition of AGT potentiates the activity of TMZ in several human tumor cell lines [6].

Current chemotherapy approaches in glioblastoma treatment are based on using high doses of TMZ (75-200 mg per square meter of body surface per day). Unfortunately, such regiment induces neurotoxicity and cognitive dysfunction expressed as a treatment-related negative side effects [25]. There is a preclinical evidence indicating that cisplatin can enhance the antitumor activity of TMZ [6].

In our study, combining of TMZ and Dox (50 nM) actions resulted in an enhancement of the anti-proliferative effect of TMZ. Besides, the anticancer activity of A24-p-TMZ complex was enhanced by $23 \%$ at its combination with the Dox.

The precise mechanism of action of TMZ in glioblastoma cells remains to be elucidated [26]. It was shown that treatment of human tumor cells with TMZ induced an increase in the activity of PARP DNA reparation enzyme involved in nucleotide excision repair. The inhibition of PARP has been reported to be a mechanism of enhancing a cytotoxicity of the methylating drug agents [6]. In this study, we showed an increased caspase-3 cleavage under the action of TMZ towards glioma cells.

A number of intracellular signaling pathways was described in the regulation of growth and survival of GBM. They include the activation of the epidermal growth factor receptor (EGFR) in these cells, and EGFR dysfunction correlates with poor prognosis in cancer patients [26]. Activation of EGFR leads to induction of downstream signaling pathways such as phosphorylation of AKT, MAPK and JNK [27].

Our data showed an increase in the level of phospho-ERK1/2 and phospho-JNK in T98G glioma cells. These kinases function downstream of the EGFR signaling which is commonly associated with GBM pathology. Thus, auto-trans-phosphorylation of EGFR could activate AP-1 via ERK1/2 and JNK
[26]. Also, ERK can regulate cell growth at the transcription level affecting an early response gene, such as c-fos, that is mediated by STAT3 (Ser727) activation. STAT3 mediates the expression of a variety of genes in response to cell stimuli, and, thus, plays a key role in many cellular processes such as growth and apoptosis. It was reported that STAT3 acts as a general positive regulator of cell transformation and oncogenesis in various tissues including brain $[28,29]$. So, STAT3 inhibition under the action of free TMZ and its form conjugated with the polymeric carrier could provide the tumor suppressor action.

Cdk1 is a catalytic subunit of highly conserved protein kinase complex known as M-phase promoting factor (MPF) which is essential for G1/S and G2/M phase transitions of the eukaryotic cell cycle. $\mathrm{pRb}$ remains phosphorylated throughout $\mathrm{S}, \mathrm{G} 2$ and $\mathrm{M}$ phases. When $\mathrm{pRb}$ is hyper-phosphorylated, it is unable to form a complex with transcription factor $\mathrm{E} 2 \mathrm{~F}$ and, therefore, to restrict a progression from the $\mathrm{G} 1$ to the $\mathrm{S}$ phase of cell cycle [30].

Thus, we found that TMZ induces apoptosis in glioma cells via activation of MAPK signaling pathway and inhibition of STAT3 and affects a transition through $\mathrm{G} 2 / \mathrm{M}$ phase. A novel polymeric carrier functionalized with the phosphatidylcholine is capable of enhancing approximately twice an anticancer therapeutic efficacy of TMZ in vitro. Combination of TMZ with the Dox (50 nM) resulted in further improvement by $23 \%$ of the anti-proliferative effect of TMZ. These features make the novel nano-formulation of TMZ a perspective candidate for further development of this drug.

\section{Acknowledgements}

The authors thank RECOOP HST Association and CSMC International Research and Innovation Management Program for support. Personal thanks are also expressed to Dr. Sandor Vari (Director, International Research and Innovation in Medicine Program, Cedars - Sinai Medical Center, Los Angeles, USA, President of the RECOOP HST Association) for inspiring collaboration between research teams involved in this work. The authors thank Professor Vadym Kavsan (Department of Biosynthesis of Nucleic Acids, Institute of Molecular Biology and Genetics, National Academy of Science of Ukraine) who was a founder of studies in the field of glioma treatment in Ukraine. 


\section{References}

1. Avgeropoulos NG, Batchelor TT. New treatment strategies for malignant gliomas. Oncologist. 1999; 4(3): 209-224.

2. Roos WP, Batista LF, Naumann SC, Wick W, Weller M, Menck CF, Kaina B. Apoptosis in malignant glioma cells triggered by the temozolomide-induced DNA lesion O6methylguanine. Oncogene. 2007; 26(2): 186-197.

3. Kanzawa T, Germano IM, Komata T, Ito H, Kondo Y, Kondo S. Role of autophagy in temozolomide-induced cytotoxicity for malignant glioma cells. Cell Death Differ. 2004; 11(4): 448-457.

4. Wedge SR, Newlands ES. O6-benzylguanine enhances the sensitivity of a glioma xenograft with low O6-alkylguanine-DNA alkyltransferase activity to temozolomide and BCNU. Br J Cancer. 1996; 73(9): 1049-1052.

5. Newlands ES, Stevens MF, Wedge SR, Wheelhouse RT, Brock C. Temozolomide: a review of its discovery, chemical properties, preclinical development and clinical trials. Cancer Treat Rev. 1997; 23(1): 35-61.

6. Friedman HS, Kerby T, Calvert H. Temozolomide and treatment of malignant glioma. Clin Cancer Res. 2000; 6(7): 2585-2597.

7. Rahman AM, Yusuf SW, Ewer MS. Anthracycline-induced cardiotoxicity and the cardiac-sparing effect of liposomal formulation. Int J Nanomedicine. 2007; 2(4): 567-583.

8. Ali I, Rahis-Uddin, Salim K, Rather MA, Wani WA, Haque A. Advances in nano drugs for cancer chemotherapy. Curr Cancer Drug Targets. 2011; 11(2): 135-146.

9. Riganti C, Voena C, Kopecka J, Corsetto PA, Montorfano G, Enrico E, Costamagna C, Rizzo AM, Ghigo D, Bosia A. Liposomeencapsulated doxorubicin reverses drug resistance by inhibiting P-glycoprotein in human cancer cells. Mol Pharm. 2011; 8(3): 683-700.

10. Shen J, He Q, Gao Y, Shi J, Li Y. Mesoporous silica nanoparticles loading doxorubicin reverse multidrug resistance: performance and mechanism. Nanoscale. 2011; 3(10): 4314-4322.

11. Riabtseva A, Mitina N, Boiko N, Garasevich S, Yanchuk I, Stoika R, Slobodyanyuk O, Zaichenko A. Structural and ColloidalChemical Characteristics of Nanosized Drug
Delivery Systems Based on Pegylated Comblike Carriers. Chem Chem Technol. 2012; 6(3): 291-295.

12. Riabtseva A, Mitina N, Grytsyna I, Boiko N, Garamus VM, Stryhanyuk H, Stoika R, Zaichenko A. Functional micelles formed by branched polymeric surfactants: Synthesis, characteristics, and application as nanoreactors and carriers. Eur Polym J. 2016; 75: 406-422.

13. Liu $\mathrm{X}, \mathrm{Zu}$ YG, Fu YJ, Yao LP, Gu CB, Wang W, Efferth T. Antimicrobial activity and cytotoxicity towards cancer cells of Melaleuca alternifolia (tea tree) oil. Eur Food Res Technol. 2009; 229(2): 247-253.

14. Heffeter P, Jakupec MA, Körner W, Chiba P, Pirker C, DornetshuberR, Elbling L, Sutterlüty H, Micksche M, Keppler BK, Berger W. Multidrugresistant cancer cells are preferential targets of the new antineoplastic lanthanum compound KP772 (FFC24). Biochem Pharmacol. 2007; 73(12): 1873-1886.

15. Sur P, Sribnick EA, Patel SJ, Ray SK, Banik NL. Dexamethasone decreases temozolomideinduced apoptosis in human gliobastoma T98G cells. Glia. 2005; 50(2): 160-167.

16. Nakagami $H$, Morishita $R$, Yamamoto $K$, Taniyama Y, Aoki M, Matsumoto K, Nakamura T, Kaneda Y, Horiuchi M, Ogihara T. Mitogenic and antiapoptotic actions of hepatocyte growth factor through ERK, STAT3, and AKT in endothelial cells. Hypertension. 2001; 37(2 Pt 2): 581-586.

17. Gartel AL, Tyner AL. The role of the cyclindependent kinase inhibitor $\mathrm{p} 21$ in apoptosis. $\mathrm{Mol}$ Cancer Ther. 2002; 1(8): 639-649.

18. Senkiv Y, Riabtseva A, Heffeter P, Boiko N, Kowol CR, Jungwith U, Shlyakhtina Y, Garasevych SG, Mitina N, Berger W, Zaichenko A, Stoika R. Enhanced anticancer activity and circumvention of resistance mechanisms by novel polymeric/ phospholipidic nanocarriers of doxorubicin. $J$ Biomed Nanotechnol. 2014; 10(7): 1369-1381.

19. Heffeter P, Riabtseva A, Senkiv Y, Kowol CR, Körner W, Jungwith U, Mitina N, Keppler BK, Konstantinova T, Yanchuk I, Stoika R, Zaichenko A, Berger W. Nanoformulation improves activity of the (pre)clinical anticancer ruthenium complex KP1019. J Biomed Nanotechnol. 2014; 10(5): 877-884. 
20. Ramge P, Unger RE, Oltrogge JB, Zenker D, Begley D, Kreuter J, Von Briesen $H$. Polysorbate-80 coating enhances uptake of polybutylcyanoacrylate (PBCA)-nanoparticles by human and bovine primary brain capillary endothelial cells. Eur J Neurosci. 2000; 12(6): 1931-1940.

21. Ochs K, Kaina B. Apoptosis induced by DNA damage O6-methylguanine is Bcl-2 and caspase-9/3 regulated and Fas/caspase-8 independent. Cancer Res. 2000; 60(20): 58155824.

22. Hickman MJ, Samson LD. Apoptotic signaling in response to a single type of DNA lesion, O(6)methylguanine. Mol Cell. 2004; 14(1): 105-116.

23. Hirose Y, Berger MS, Pieper RO. Abrogation of the Chk1-mediated G(2) checkpoint pathway potentiates temozolomide-induced toxicity in a p53-independent manner in human glioblastoma cells. Cancer Res. 2001; 61(15): 5843-5849.

24. Günther W, Pawlak E, Damasceno R, Arnold H, Terzis AJ. Temozolomide induces apoptosis and senescence in glioma cells cultured as multicellular spheroids. $\mathrm{Br} \mathrm{J}$ Cancer. 2003; 88(3): 463-469.
25. Munoz JL, Rodriguez-Cruz V, Greco SJ, Ramkissoon SH, Ligon KL, Rameshwar P. Temozolomide resistance in glioblastoma cells occurs partly through epidermal growth factor receptor-mediated induction of connexin 43. Cell Death Dis. 2014; 5: e 1145.

26. Meng Q, Xia Y. c-Jun, at the crossroad of the signaling network. Protein Cell. 2011; 2(11): 889-898.

27. Schlessinger K, Levy DE. Malignant transformation but not normal cell growth depends on signal transducer and activator of transcription 3. Cancer Res. 2005; 65(13): 58285834.

28. Chiarle R, Simmons WJ, Cai H, Dhall G, Zamo A, Raz R, Karras JG, Levy DE, Inghirami G. Stat3 is required for ALK-mediated lymphomagenesis and provides a possible therapeutic target. Nat Med. 2005; 11(6): 623-629.

29. Korenjak M, Brehm A. E2F-Rb complexes regulating transcription of genes important for differentiation and development. Curr Opin Genet Dev. 2005; 15(5): 520-527.

Received 25.03.2016 